\title{
Severe respiratory adverse events associated with rituximab infusion
}

\author{
Koichi Kamei • Shuichi Ito $\cdot$ Kazumoto Iijima
}

Received: 17 October 2009 / Accepted: 26 October 2009/Published online: 19 December 2009

(C) IPNA 2009

Sirs,

We read with great interest the article in Pediatric Nephrology entitled "Fatal pulmonary fibrosis after rituximab administration" by Chaumais et al. [1]. We believe that patients who have lung complications are at an extremely high risk of respiratory adverse events after rituximab infusion. Respiratory events, such as cough, bronchospasm, and dyspnea, are relatively common adverse effects of rituximab. At our center, 28 patients have received rituximab to date (21 with steroid-dependent nephrotic syndrome and 7 with steroid-resistant nephrotic syndrome). Ten (36\%) of these patients have experienced respiratory events, such as an infusion reaction. Several case reports have described severe respiratory injuries associated with the use of rituximab, such as interstitial pneumonitis and acute respiratory distress syndrome, although the precise frequency is unknown.

There have been 32 reports (total of 62 cases) of rituximabinduced severe respiratory adverse events [2]. In these reports, elderly people were considered to be at high risk, and most of the patients were older than 50 years (median age 62 years, range 9-88). Of these 62 patients (85\%), 53 had lymphoma or B-cell lymphocytic leukemia, and 46 (74\%) suffered from interstitial pneumonitis and other complications, including bronchiolitis obliterans with or without organizing pneumonia (4 patients), acute respiratory distress

K. Kamei $(\bowtie) \cdot$ S. Ito

Department of Nephrology,

National Center for Child Health and Development,

2-10-1 Okura,

Setagaya-ku, Tokyo 180-8535, Japan

e-mail: kamei-k@ncchd.go.jp

\section{K. Iijima}

Department of Pediatrics,

Kobe University Graduate School of Medicine,

7-5-1 Kusunoki-cho,

Chuo-ku, Kobe City, Japan syndrome (3), organizing pneumonia (3), hypersensitivity pneumonia (2), and pulmonary fibrosis (1); for three patients, there was no definite diagnosis. Prompt initiation of steroid therapy appears to have conferred benefit, although some cases resulted in fatal outcomes (12 patients, 19\%).

Most of the patients reported were elderly, but there were two pediatric patients and, surprisingly, both of these children suffered from refractory nephrotic syndrome [1,2]. Bitzan et al. reported a 14-year-old boy who suffered frequent relapsing nephrotic syndrome despite the use of various immunosuppressants [2]. Respiratory symptoms (cough, chest pain, fatigue, and fever) occurred 19 days after the last infusion of rituximab, and a chest computed tomography demonstrated the presence of diffuse, groundglass infiltrates in both lungs, which was compatible with interstitial pneumonitis. The patient's respiratory symptoms improved without recurrence. However, he was successfully treated with prednisolone and antibiotics.

It may be interesting to investigate whether pediatric patients with nephrotic syndrome have a high risk of interstitial pneumonitis. At the present time, we recommend that a routine chest radiograph is obtained before riuximab infusion is initiated. We believe that clinicians should be highly alert to the possibility of severe respiratory adverse events and should never administer rituximab to patients already suffering from lung disease, such as pleural effusion, pneumonia, and atelectasis.

\section{References}

1. Chaumais MC, Garnier A, Chalard F, Peuchmaur M, Dauger S, Jacqz-Agrain E, Deschênes G (2009) Fatal pulmonary fibrosis after rituximab administration. Pediatr Nephrol 24:1753-1755

2. Bitzan M, Anselmo M, Carpineta L (2009) Rituximab (B-cell depleting antibody) associated lung injury (RALI): a pediatric case and systematic review of the literature. Pediatr Pulmonol 44:922-934 